\title{
Visual experiences during paralysis
}

\section{Emma M. Whitham ${ }^{1}$, Sean P. Fitzgibbon ${ }^{1}$, Trent W. Lewis ${ }^{2}$, Kenneth J. Pope ${ }^{2}$, Dylan DeLosAngeles ${ }^{1}$, C. Richard Clark ${ }^{3}$, Peter Lillie ${ }^{4}$, Andrew Hardy ${ }^{4}$, Simon C. Gandevia ${ }^{5}$ and John O. Willoughby ${ }^{1 *}$}

\author{
Department of Neurology, Centre for Neuroscience, Flinders University and Medical Centre, Adelaide, SA, Australia \\ 2 School of Computer Science, Engineering and Mathematics, Flinders University, Adelaide, SA, Australia \\ ${ }^{3}$ Cognitive Neuroscience Laboratory, School of Psychology, Flinders University, Adelaide, SA, Australia \\ ${ }^{4}$ Department of Anaesthesia and Intensive Care, Flinders Medical Centre, Adelaide, SA, Australia \\ ${ }^{5}$ Neuroscience Research Australia, University of New South Wales, Sydney, NSW, Australia
}

\section{Edited by:}

Olivier Bertrand, INSERM, France

\section{Reviewed by:}

Olivier Bertrand, INSERM, France Robert D. Rafal, University of Wales, UK

\section{*Correspondence:}

John O. Willoughby, Department of Neurology, Flinders University and Medical Centre, PO Box 2100, Adelaide, SA 5001, Australia. e-mail: john.willoughby@flinders. edu.au

\begin{abstract}
Rationale: Paralyzed human volunteers $(n=6)$ participated in several studies the primary one of which required full neuromuscular paralysis while awake. After the primary experiment, while still paralyzed and awake, subjects undertook studies of humor and of attempted eye-movement. The attempted eye-movements tested a central, intentional component to one's internal visual model and are the subject of this report. Methods: Subjects reclined in a supportive chair and were ventilated after paralysis (cisatracurium, $20 \mathrm{mg}$ intravenously). In illumination, subjects were requested to focus alternately on the faces of investigators standing on the left and the right within peripheral vision. In darkness, subjects were instructed to look away from a point source of light. Subjects were to report their experiences after reversal of paralysis. Results: During attempted eye-movement in illumination, one subject had an illusion of environmental movement but four subjects perceived faces as clearly as if they were in central vision. In darkness, four subjects reported movement of the target light in the direction of attempted eye-movements and three could control the movement of the light at will. Conclusion: The hypothesis that internal visual models receive intended ocular-movement-information directly from oculomotor centers is strengthened by this evidence.
\end{abstract}

Keywords: neuromuscular block, perception, efference copy, oculomotor copy

\section{INTRODUCTION}

Visual information provides the major input to one's vision of the visible environment. However, it is known that information about eye position is also essential in the construction of one's vision of the world. Non-retinal contributions about eye position include proprioceptive input from oculomotor muscles and information direct from oculomotor gaze centers (Donaldson, 2000). As reviewed by Donaldson (2000), a central nervous system source of knowledge about eye or limb position and movement has been hypothesized for many years, labeled "corollary discharge" by Sperry (1950) and "efference copy" by von Holst and Mittelstaedt (1950). Evidence for an efference copy has been obtained from studies in paralyzed animals in total darkness. In these, activity in the nucleus of the optic tract continued, and was time-locked with increased activity in oculomotor neurons (Bon et al., 1984), implying that central neurons driving eye-movements also directly activate neurons responsive to retinal movement. Furthermore, in behaving monkeys, a pathway from superior colliculus via the medio-dorsal thalamus to the frontal eye field conveying a corollary discharge for coordinating sequential saccades and possibly for stabilizing vision across saccades has been described by Sommer and Wurtz (2004).

In this report we use the term "oculomotor copy" to differentiate the concept from somato-motor efference copy. We use the term "visual model" simply to refer to the phenomenology of the experience of our external world, not-withstanding the strong criticism of such use by O'Regan and Noe (2001). They propose that there is no visual model. Instead, "Visual perception can be understood as the activity of exploring the environment in ways mediated by knowledge of the relevant sensorimotor contingencies... [and of being] capable of exercising mastery of vision-related rules of sensorimotor contingency."

Evidence of an oculomotor copy is provided by movement of the visual model in the same direction as shifts of voluntary gaze in subjects with complete bilateral extra-ocular muscle paralysis. Full paralysis ensures no change in the visual stimulus and no proprioceptive change from either eye during attempted shifts of gaze. Suggestive findings, namely relocation of the visual scene (the visual model) have been reported in one fully paralyzed subject in good illumination (Stevens et al., 1976). The description of the experience during full paralysis was not straight forward, with displacement of the visual environment being described without movement and as "not necessarily visual in nature." Results that suggest the outcome might be different in darkness come from another report from the same group (Matin et al., 1982) in which movement of a visual target, a light, was observed during various levels of generalized partial paralysis in darkness but not in illumination. In the experiment described below, we had an opportunity to study six subjects during complete pharmacologically induced paralysis. during which they activated oculomotor commands in illumination and in darkness, further testing the hypothesis of a direct oculomotor influence on one's visual model. 
The opportunity for this experiment arose subsequent to studies of scalp electrical recordings in paralyzed human volunteers which evaluated the extent of contamination of the electroencephalogram (EEG) by electromyographic artifact (Whitham et al., 2007, 2008; Pope et al., 2009). After completion of the EEG studies and for a few minutes before subjects were sedated with propofol for the recovery from paralysis, subjects participated in studies of humor and attempted eye-movement. Experiences of the visual tasks are the subject of this paper.

Reports of conscious experiences of paralysis date from 1947, with the advent of purified preparations of D-tubocurare suitable for neuromuscular blockade (Smith et al., 1947). At that time questions were raised regarding the potential of these agents to produce central depressant effects. Experiments undertaken in awake-paralyzed subjects revealed that there was no effect on the paralyzed subjects' memory or cognitive functioning (Smith et al., 1947). These experiments have since been repeated and similar findings made for both curare and succinylcholine (Stevens et al., 1976; Messner et al., 2003).

\section{EXPERIMENTAL PROCEDURE}

The paralysis of EEG Research Unit staff and other volunteers was approved by the Flinders Clinical Research Ethics Committee. All participants signed written informed consent. No individuals were approached to participate in the paralysis experiments; all subjects actively sought participation in the study.

The adult subjects undergoing paralysis $(n=6$, one female) were familiar with the scientific background and purpose of the EEG study and agreed to participate in the additional experiential tasks. None was taking medications likely to affect CNS function. Three subjects had migraine without aura (approximately 12-24 per annum), one of whom also had an un-medicated dysthymic disorder and who was also a meditator.

\section{THE EXPERIMENTAL SETTING}

The primary study preceding the visual experiments required paralysis and is described in full elsewhere (Whitham et al., 2007). In brief, the experiment tested the extent to which electromyography (EMG) contaminates scalp electrical recording. To this end, scalp electrical activity was recorded before and after complete neuromuscular paralysis in awake volunteers. Recordings were made in a room-sized Faraday cage in an EEG laboratory. The subjects were seated on a chair with legs elevated, in a supported position that required no change in body posture after paralysis. Ventilator and monitoring equipment were located outside the cage and the existing Faraday cage ports were used for lines and airways. A neurologist and one anesthetist remained in the cage throughout the experiment. A second anesthetist remained outside the cage to control the ventilator and to undertake monitoring. The entire procedure was rehearsed until the protocol ran without error. The paralyzant was cisatracurium $20 \mathrm{mg}$ given by intravenous injection. Blood pressure, expired $\mathrm{CO}_{2}$, finger oxygen saturation, EEG, ECG, and respiratory movements were all monitored. Muscular paralysis was evaluated by right common peroneal nerve stimulation to extensor digitorum brevis. The compound muscle action potential was fully abolished about $5 \mathrm{~min}$ after administration of paralyzant and cranial, including peri-ocular
EMG as well as electrooculogram (EOG) activity, remained absent for the duration of the studies.

After the primary EEG experiment had been completed, the experiential experiments began. Scalp recording continued throughout, so providing continuing evidence of ocular muscle paralysis. However, possible EEG correlation with the visual tasks has not been attempted.

\section{VISUAL TASKS}

The experimental interventions were verbal requests to make saccadic eye-movements in two lighting conditions. Subjects were not given prior knowledge of the experiences of subjects previously studied. In each visual condition, investigators elevated both eyelids with a soft probe to enable vision. First, in an illuminated environment, subjects were instructed to focus alternately on the faces of investigators standing on the left and the right side of the subject but within peripheral vision, i.e., they attempted eye-movement by left/right attentional shift. Second, with light eliminated from within the Faraday cage, subjects were instructed to attempt to look away from the point source of light from a light-emitting diode of $2 \mathrm{~mm}$ diameter held approximately $1 \mathrm{~m}$ from the paralyzed subject in a position expected to be close to foveal vision for the paralyzed eyes. The subject was instructed to attempt horizontal or vertical eye-movements around the light and to note any movement of the point of light which accompanied the left/right attentional shift. In view of the reports from the first three participants, two tasks were added for completion by the remaining participants. One task carried out under illumination was to attempt to read a sentence printed in large and easily readable text, while the displayed sentence was held in the expected foveal visual field. The second, in darkness, was to focus alternately on the investigators they remembered as standing on the left and the right side of the subject. In all experiments, absence of activity in EMG and EOG recordings was used to confirmed absence of ocular movement. Subjects were not given any expectation of what they might experience but knew they would be asked if there was any movement of the light. The subjects were to report the results after reversal of paralysis.

Following completion of the visual tasks, and the humorrelated task not presented here, the subjects were sedated with intravenous propofol and the paralysis was allowed to reverse, assisted by intravenous neostigmine. Reversal of paralysis and reawakening was complete after about $40 \mathrm{~min}$ and a detailed interview of the experiences during paralysis was undertaken.

Subsequent to this experiment, one subject had pre-surgical orbital infiltration of local anesthetic agent, resulting in complete paralysis of one eye. This provided an opportunity to compare visual perception during attempted movement with one paralyzed eye in a subject previously studied during bilateral paralysis. The expectation from Stevens et al. (1976), also derived from monocular paralysis in one subject, is that non-continuous displacement of the visual model would occur, presumptively driven by positional information from the intact eye. We repeated their experiment.

\section{RESULTS}

All subjects tolerated the procedure without report of adverse effects and they reported feelings of alertness and curiosity. They 
felt physically relaxed and comfortable during paralysis and there were no observed changes in posture or facial expression due to paralysis. Cranial EMG activity and EOG activity were absent throughout the experiments.

In all subjects, bilateral eye opening resulted in vision that seemed normal. There was no diplopia or blurring, except in the one subject whose contact lenses moved during the study. One subject (F, Table 1) reported partial fading of areas of vision. In all, these are consistent with there being no change in the resting position of the eyes and no changes in accommodation. Two types of illusory experience were reported during attempts to shift gaze: detailed vision (illusion of detail, Figure 1) and movement of the visual field (illusion of movement, Figure 2), both in the direction of attempted gaze. Hand-drawn depictions of these illusions

Table 1 | Reports of experiences in six subjects in two lighting conditions.

\begin{tabular}{lll}
\hline Subject & $\begin{array}{l}\text { Visual illusions in } \\
\text { illumination: left/right } \\
\text { attentional shift }\end{array}$ & $\begin{array}{l}\text { Visual illusions in } \\
\text { darkness: left/right } \\
\text { attentional shift }\end{array}$ \\
\hline A & Detail & Movement \\
B & Detail & Movement \\
C & Detail & No movement \\
D & Movement & Movement \\
E & Detail & Movement \\
F (female) & Neither movement nor detail & No movement
\end{tabular}

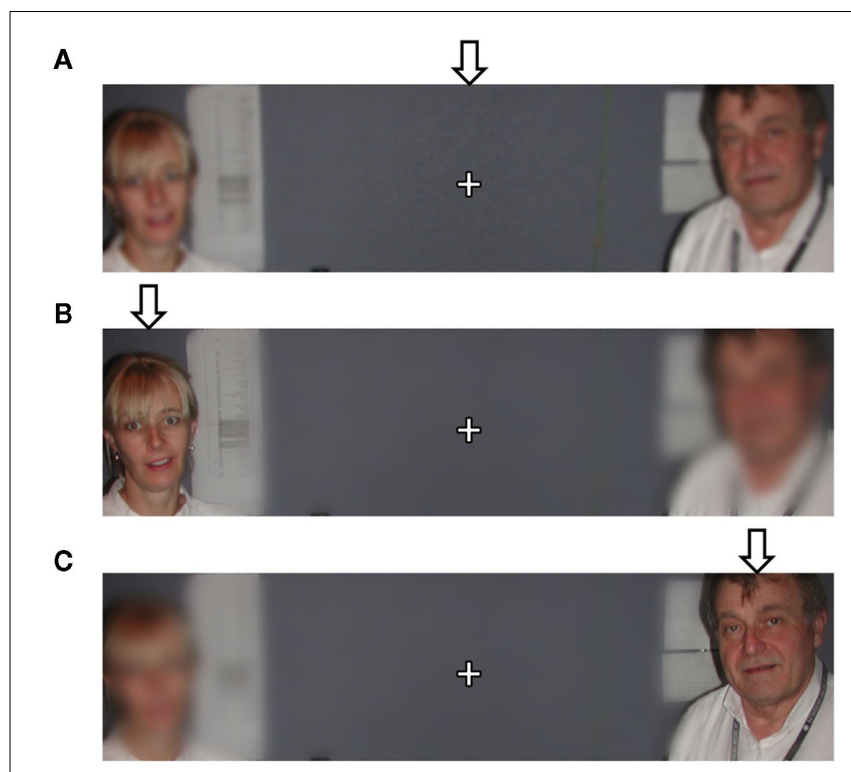

FIGURE 1 | Static depiction of the illusion of detail with illumination during paralysis. (A) When attending to the center of the foveal field (indicated by + ) subjects report poor detail of faces in the left and right periphery. When attending to either the left (B) or right (C) face (attempted gaze without actual movement of the foveal field due to paralysis) subjects report increased detail of the attended face. The arrows indicate the position of the attempted gaze. The supplementary material shows an animation of this depiction. are illustrated in the figures and can be seen in a Movie S1 in Supplementary Material.

In an illuminated environment with binocular vision, five subjects had no illusion of movement of the environment with attempted lateral movements (Table 1). Of the five subjects with no illusion of movement, four experienced the target faces as clear, apparently as clear as if being viewed directly, i.e., an illusion of detail (Table 1, Figure 1, and Supplementary Material).

To test if the impression of clear vision in the attended region of the visual field was as normal as claimed, subjects in the last three studies were asked to read an unfamiliar sentence, the center of which was held in the predicted foveal position. One subject could read only a few centrally located words, that is, the subject did not have good visual acuity away from the foveal region. In another subject, a contact lens was displaced, making the task invalid and in another, with no illusion of clarity on lateral gaze, none of the text was legible. One subject (D), however, described the environment moving fully and in the direction of attempted gaze, i.e., an illusion of movement (Supplementary Material).

In darkness, with a 2-mm target light close to foveal vision, three of six subjects reported that eye-movement attempts in to the left and right from the resting position were accompanied by movement of the target light in the direction of attempted movement (Figure 2 and Supplementary Material). None of the subjects experiencing movement of the target light in darkness reported disappearance or fading of the light during movement: it remained bright.

In one other subject (E), the task of attempting to look at the faces (in darkness) to left and right, evoked movement of the target light, but not the task of looking to left and right away from the light. In three subjects $(\mathrm{A}, \mathrm{B}, \mathrm{D})$, the extent of apparent movement

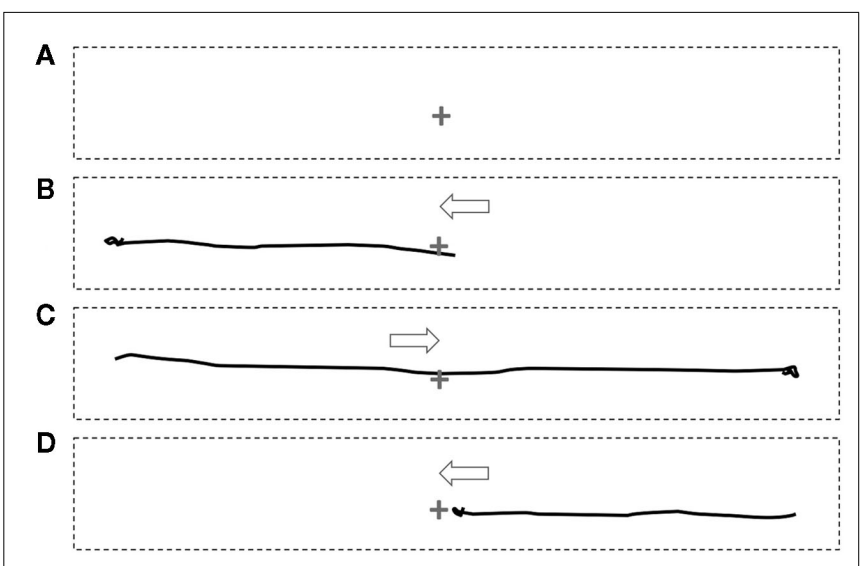

FIGURE 2 | Static depiction of the illusion of movement in darkness during paralysis. Subjects were presented with a point of light (indicate by $+)$ in the center of the foveal field surrounded by darkness. When attempting to gaze away from the light (without actual movement of the foveal field due to paralysis), subjects report movement of the light with a trajectory consistent with the direction of attempted gaze. The perceptions were, consecutively: rest (A), attempted gaze to left (B), attempted gaze to right (C), and return (D). The solid black lines depict the trajectory of the illusory movement of the point of light. The arrows indicated the direction of the attempted gaze. The supplementary material shows an animation of this depiction. 
of the target appeared to match the extent of attempted movement. The impression of movement of the target was that it was not entirely smooth, having a slight tremulous or jerky quality and with slight over-shoots. Motivated by the ease of movement of the light with attempted shifts of attention, three subjects found they were able to draw simple figures using attentional shifts and the image of the light: circles, triangles, squares, ovals, and an Archimedes's spiral (hand-drawn depictions in Figure 3 and Supplementary Material).

A signature proved difficult, the light appearing to jerk well beyond the small excursion needed for small letters. Two subjects reported no movement of the target in any task or condition.

In illumination, subject A, later having pre-surgical monocular paralysis, had experienced no illusion of movement with an illusion of detail when fully paralyzed. In contrast, with paralysis of one eye and the un-paralyzed eye covered, attempted movement of the paralyzed eye reliably resulted in movement of the visual environment in the direction of attempted movement, rather than simply the illusion of detail experienced during bilateral paralysis.

\section{DISCUSSION \\ PARALYSIS}

The experience of paralysis is unpleasant when it occurs during unintended wakefulness with surgical procedures or during therapeutic paralysis (Moerman et al., 1993; Wagner et al., 1998). The experiences of experimental awake-paralysis subjects clearly differ significantly from those who experience unplanned wakefulness during surgery. Horror, fear, and often pain are described in these situations in which the expectation is to remain unaware of a usually painful event, and wakeful paralysis during therapeutic paralysis is associated with feelings of fear, powerlessness, and helplessness (Moerman et al., 1993; Wagner et al., 1998). Our study confirms previous findings that, when planned, wakeful paralysis

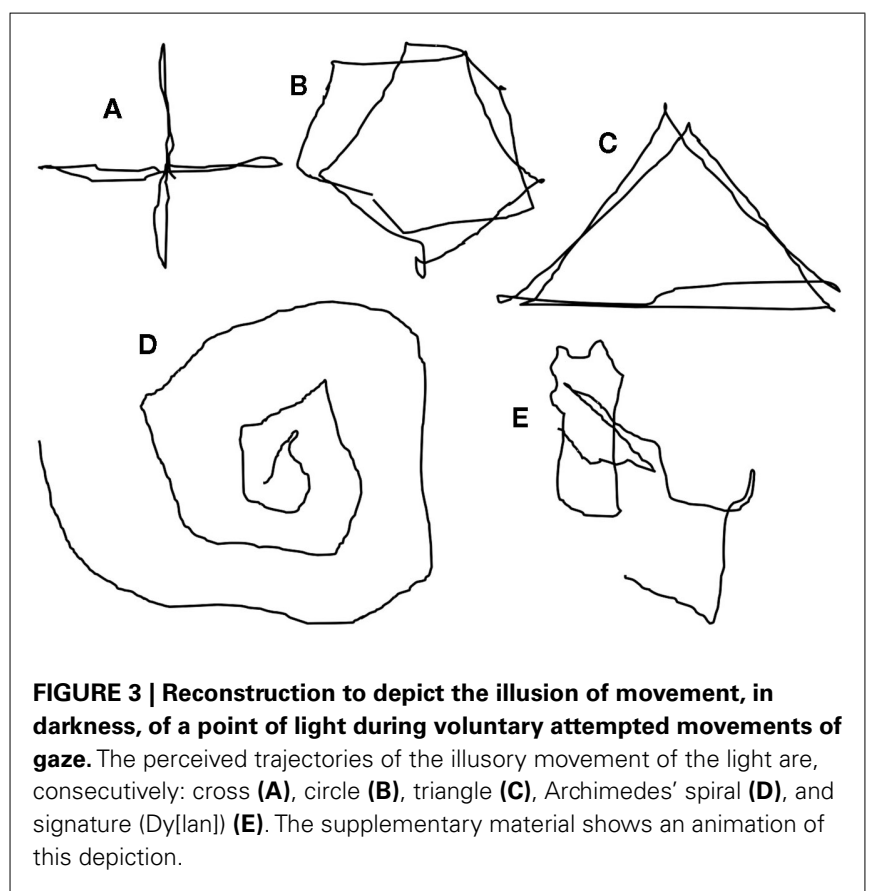

with adequate ventilation is in itself a pleasant experience (Smith et al., 1947; Gandevia et al., 1993; Topulos et al., 1993).

\section{VISUAL EXPERIENCES - ILLUSION OF MOVEMENT}

One's visual model of the three dimensional world depends both on visual input and on the position of the eyes (Figure 4). Information about eye position, independent of visual input, likely comes from proprioceptive input from extra-ocular muscles (Skavenski, 1972; Donaldson, 2000) as well as from a centrally instantiated construct of "where one is looking." The central mechanism is hypothesized to involve motor command signals from higher oculomotor or gaze centers, whereby a copy of the oculomotor command provides "sensory" information about eye position (Sperry's corollary discharge), or an expectation of where the eyes, and perhaps visual field, will be after an eye-movement (von Holst and Mittelstaedt, 1950).

The experiences of the paralyzed subjects provide strong support for the importance of an oculomotor copy in determining the visual model. Although the exception in an illuminated environment (one of six subjects), rotation of the visual environment occurred in the direction of attempted saccades in darkness. The subject with rotation in illumination might have acquired enhanced attentional strength through years of practising meditation, so that enhanced, attentionally driven, oculomotor information may be explanatory in this individual: the result is consistent with the oculomotor copy being dominant over the competing, unchanging visual and ocular proprioceptive input. The majority experience of no movement in illumination (five of six subjects) is consistent with strong, stationary, visual, and proprioceptive inputs overriding the oculomotor copy.

It is of relevance that one subject who had an illusion of shifting detail but no movement when fully paralyzed in illumination, experienced smooth movement of the environment in the direction of gaze during monocular paralysis, in partial agreement with

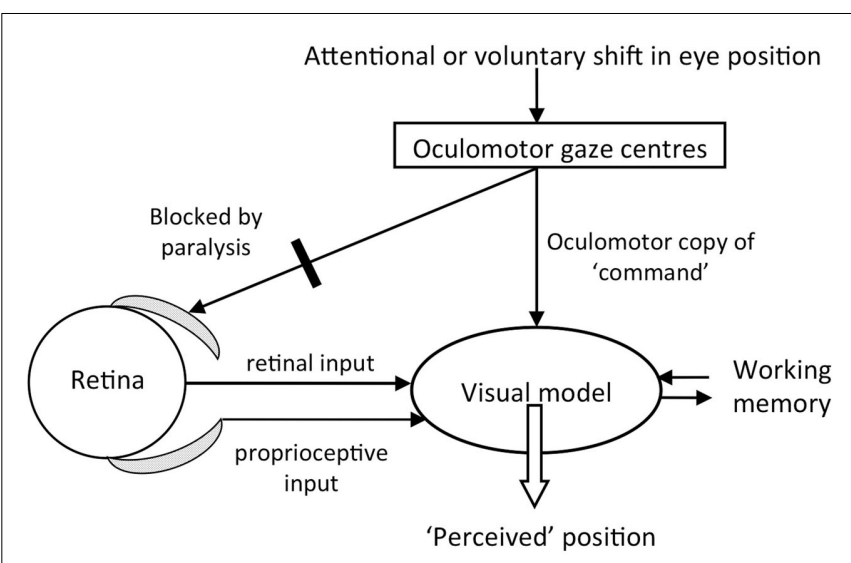

FIGURE 4 | Diagram of the visual model with hypothesized inputs. In this study, blockade by cisatracurium of ocular muscles left proprioception unchanged and visual input stationary. We varied the strength of visual input by using illumination and darkness, and varied oculomotor center activity by requesting attentional or voluntary eye-movements. The reported experiences indicated that the "oculomotor copy" was able to bias the construction of the visual model, especially when illumination was diminished. 
one report (Stevens et al., 1976) discussed above, and counter to one other (Brindley et al., 1976). These experiences are consistent with proprioception from the covered-but-intact eye providing strong positional information during monocular paralysis causing the visual model to move, in contrast to the absence of proprioceptive change during bilateral paralysis. The findings emphasize the different consequences putatively due to different proprioceptive information.

In darkness, movement of the visual environment in the direction of the attempted saccades was the majority experience (four of six). In one of these four subjects however, it was only the attempt to attend to persons known to be present to the left and right that resulted in movement of the visual model, not simply the obeying of an instruction to move focus away from the light. In three others, movement of the light occurred when-ever attempting to focus away from the light and was so easily effected that the individuals attempted to use the light "as chalk on a board" to construct figures and even to try a signature, powerful evidence for an oculomotor copy. Thus, in four subjects, the experiences are consistent with the speculations of Bays and Husain (2007) that other features in the visual field are important in determining the visual model in normal conditions. The oculomotor copy dominates over a stationary but much reduced visual input, as well as an unchanged proprioceptive input, in our subjects. In the subjects not experiencing movement of the visual environment in darkness, either a strong memory of their visual world, or unchanged proprioceptive inputs can be hypothesized to have "fixed" their visual model.

While there is evidence that both retinal (Thilo et al., 2004) and central mechanisms (Bridgeman, 1981; Niemeier et al., 2003) appear to participate in saccadic suppression, suppression was not a noticeable feature of the fictive saccades in our study. This observation diminishes the significance of centrally mediated saccadic suppression if the saccades are made without retinal movement and, therefore, without visual blurring.

Movement of the visual model in illumination (one subject) and in darkness (four subjects) are an advance on what was previously observed in subjects during paralysis. Brindley et al. (1976) used local agents to block movement and sensation in one eye and did not observe movement of the visual model in illumination. Stevens et al. (1976) distinguished between displacement (sudden shifts of the visual model without moving imagery) and movement (continuous movement of a visual model) and they observed both in varying degrees of paralysis, in illumination. Displacement, rather than movement, seemed to be what occurred with full paralysis. Consistent with this, we have shown illusory movements during an attempted saccade to be infrequent in illumination (one subject). However, movement of the visual model was the usual experience in darkness in our study (four subjects). Changed visual input in darkness versus illumination is an obvious explanatory factor for different perceptions reported. Another possible influence on the visual model could be the level of a sense of security of the paralyzed subjects. In the present study, fully paralyzed subjects were at all times comfortable and psychologically relaxed and had been so for approximately $20 \mathrm{~min}$ of paralysis. Ventilation was via a laryngeal mask airway, the insertion of which was well-tolerated. This recent technique is more comfortable than methods available in previous studies. In our unaroused subjects, construction of perceptions may have been more natural than in situations of spontaneous breathing under curare-induced partial paralysis. Whatever the explanation, the majority of our subjects described robust movement of visual models during paralysis, perceptions that point to non-retinal, non-proprioceptive, central oculomotor copy information being a significant contributor to perceived eye position, occasionally in illumination, and usually in darkness. The visual findings parallel motor findings in subjects attempting limb movement with de-efferented and de-afferented ischemic limbs. In these, there was also an illusion of displacement in the intended direction, which led to markedly inaccurate estimates of limb position (Gandevia et al., 2006; Walsh et al., 2010). The size of the illusory displacement increased when the size of the motor command was increased voluntarily.

Manipulations of visual processing were simpler in this study of full paralysis than in the partial paralysis experiments of Matin et al. (1982) especially with the possible limitations of subjectcomfort discussed above. In our study the visual stimuli and proprioceptive inputs to the visual model were fixed in each condition, and importantly, our light was positioned in the estimated foveal field; so the conclusions do not relate to visual eccentricity of the target nor of altered visual or proprioceptive input. In darkness, our observers saw no spontaneous movement of the light in contrast to Matin and colleagues' subjects who observed drift of the light downwards or upwards. We propose that in Matin and colleagues, attention to an eccentrically positioned target light induced an oculomotor drive, and copy, that in turn led to amplified eccentric positioning of the target light in their subjects' visual model. Even so, the reports of Matin and colleagues of displaced positioning of the visual target are also consistent with the oculomotor copy being an important aspect of visual modeling, which we support with this data.

Thus, during full paralysis, evidence for an oculomotor copy can sometimes be revealed in illumination, and is frequently revealed in darkness. Our findings greatly extend, and do not contradict, the limited findings in previous reports addressing the question of the oculomotor copy. As first suggested by Bridgeman (1981) and as reviewed more recently by Feldman (2009) and Bays and Husain (2007), the hypothesized function of the oculomotor copy has shifted away from its importance in maintaining a stable visual model during exploratory saccades, to being one of many components of a visuo-motor control system. Niemeier et al. (2003) propose that in making saccades to a new target, the brain optimizes available imperfect inputs from the visual scene, from retinal movement detectors, and from eye position, the latter due to both ocular muscle proprioceptors and an oculomotor copy. Feldman (2009) suggests a threshold control theory, invoking a centrally generated pattern of excitatory thresholds for the appropriate set of (oculo)motor neurons so as to achieve a particular ocular position. The role of an oculomotor copy in this proposal might be its slight contribution to common spatial frames of reference for eye-movement and perception.

Alternatively, in keeping with the O'Regan and Noe (2001) proposal eliminating the visual model, the ability to move the light arises from a subject applying expertise in visuo-motor exploration to look in one direction, without actually effecting any eyemovement. Expertise with visuo-motor exploration would require 
the light be displaced in the opposite direction and, because the light is still in the center of vision, it is erroneously placed instead the direction of attention. If this correctly interprets the O'Regan and Noe proposal, the experiment does not seem to require an oculomotor copy. However, there are pathways connecting motor and visual centers (Sommer and Wurtz, 2004), which could contribute either to expertise in visuo-motor exploration, or to an oculomotor copy.

\section{VISUAL EXPERIENCES - ILLUSION OF DETAIL}

Of interest in subjects whose visual models remained unmoved in normal illumination (five of six), the majority experience (in four) was that the targets of attention of the attempted saccades were perceived with normal clarity. The perceived normal detail of these familiar faces appears to be illusory, in view of one subject's inability to read words away from the foveal position. Nevertheless, the experiences support the view that visual attention is associated with enhanced processing of the attended object. It is well known that visual attention can be dissociated from foveal vision. Recent studies present functional magnetic resonance imaging evidence of activity in the eye-fields and in the superior parietal lobule in the decoupling, and of activity in the precentral sulcus and posterior parietal cortex during maintained non-foveal attention (Kelley et al., 2008). We hypothesize that enhanced processing of peripheral visual input and memory of the target faces enabled illusory depiction of facial detail.

\section{ABSENCE OF VISUAL FADING}

In this experiment the observers were artificially ventilated without cranial fixation. The visual targets were human faces or a handheld light, neither of which can have been fully immobile. Together, movement of the head or of the targets likely accounts for the

\section{REFERENCES}

Bays, P. M., and Husain, M. (2007). Spatial remapping of the visual world across saccades. Neuroreport 18, 1207-1213.

Bon, L., Corazza, R., and Inchingolo, P. (1984). Oculomotor corollary discharge in the cat pretectum. Arch. Int. Physiol. Biochim. 92, 153-157.

Bridgeman, B. (1981). Cognitive factors in subjective stabilization of the visual world. Acta Psychol. (Amst.) 48, 111-121.

Brindley, G., Goodwin, G. M., Kulikowski, J. J., and Leighton, D. (1976). Stability of vision with a paralysed eye. J. Physiol. (Lond.) 258, 65p-66p.

Donaldson, I. M. L. (2000). The functions of the proprioceptors of eye muscles. Philos. Trans. R. Soc. Lond. B Biol. Sci. 355, 1685-1754.

Feldman, A. G. (2009). New insights into action-perception coupling. Exp. Brain Res. 194, 39-58.

Gandevia, S. C., Killian, K., McKenzie, D. K., Crawford, M., Allen, G. M., Gorman, R. B., and Hales, J.

absence of visual fading (Martinez-Conde et al., 2006) in five of six subjects, with only slight fading in one.

\section{CONCLUSION}

In a serendipitous experimental situation, during which there was complete neuromuscular paralysis and its attendant absence of eye-movement, retinal change, and proprioceptive input, we demonstrated that there was striking, controllable movement of the visual environment during attempted shifts of gaze. This evidence provides the strongest support so far of an oculomotor command copy directly influencing one's internal visual model, i.e., intention competes with actual eye position to determine the visual model. It is also consistent with previous findings of a direct motor input in the internal model of limb position.

\section{ACKNOWLEDGMENTS}

The EEG Research Unit was funded by an equipment grant from The Wellcome Trust, London, UK. Project support has been provided by the National Health and Medical Research Council and Flinders Medical Centre Foundation. UCB Pharma and Pfizer provided fellowship support to Dr. Whitham. We also thank our colleagues Dr. Bry Wyman, University of Wisconsin, Dr. Arduino Mangoni and Ms. Anne-Louise Smith, Flinders University, and Dr. Rob Wilcox, Flinders Medical Centre, for their participation as subjects in the paralysis experiments. Mr. Steven Loveless, Mr. Rik Fronsko, and Ms. Alyson Pulbrook provided technical assistance.

\section{SUPPLEMENTARY MATERIAL}

The Movie S1 for this article can be found online at http://www. frontiersin.org/human_neuroscience/10.3389/fnhum.2011.00160/ abstract

(2003). The bispectral index declines during neuromuscular block in fully awake persons. Anesth. Analg. 97, 488-491.

Moerman, N., Bonke, B., and Oosting, J. (1993). Awareness and recall during general anesthesia. Facts and feelings. Anesthesiology 79, 454-464.

Niemeier, M., Crawford, J. D., and Tweed, D. B. (2003). Optimal transsaccadic integration explains distorted spatial perception. Nature 422, 76-80.

O'Regan, J. K., and Noe, A. (2001). A sensorimotor account of vision and visual consciousness. Behav. Brain Sci. 24, 939-973; discussion 973-1031.

Pope, K. J., Fitzgibbon, S. P., Lewis, T. W., Whitham, E. M., and Willoughby, J. O. (2009). Relation of gamma oscillations in scalp recordings to muscular activity. Brain Topogr. 22, 13-17.

Skavenski, A. A. (1972). Inflow as a source of extraretinal eye position information. Vision Res. 12, 221-229.
Smith, S. M., Brown, H. O., Toman, J. E. P., and Goodman, L. S. (1947). The lack of cerebral effects of d-tubocurarine. Anesthesiology 8 , $1-13$.

Sommer, M. A., and Wurtz, R. H. (2004). What the brain stem tells the frontal cortex. II. Role of the SC-MD-FEF pathway in corollary discharge. J. Neurophysiol. 91, 1403-1423.

Sperry, R. W. (1950). Neural basis of the spontaneous optokinetic response produced by visual inversion. J. Comp. Physiol. Psychol. 43, 482-489.

Stevens, J. K., Emerson, R. C., Gerstein, G. L., Kallos, T., Neufeld, G. R., Nichols, C. W., and Rosenquist, A. C. (1976). Paralysis of the awake human: visual perceptions. Vision Res. 16, 93-98.

Thilo, K. V., Santoro, L., Walsh, V., and Blakemore, C. (2004). The site of saccadic suppression. Nat. Neurosci. 7, 13-14.

Topulos, G. P., Lansing, R. W., and Banzett, R. B. (1993). The experience 
of complete neuromuscular blockade in awake humans. J. Clin. Anesth. 5, 369-374.

von Holst, E., and Mittelstaedt, $\mathrm{H}$. (1950). Das Reafferenzprinzip (Wechselwirkungen zwischen Zentralnervensystem und Peripherie). Naturwissenschaften 37, 464-476.

Wagner, B. K., Zavotsky, K. E., Sweeney, J. B., Palmeri, B. A., and Hammond, J. S. (1998). Patient recall of therapeutic paralysis in a surgical critical care unit. Pharmacotherapy 18, 358-363.

Walsh, L. D., Gandevia, S. C., and Taylor, J. L. (2010). Illusory movements of a phantom hand grade with the duration and magnitude of motor commands. J. Physiol. (Lond.) 588, 1269-1280.

Whitham, E. M., Lewis, T., Pope, K. J., Fitzgibbon, S. P., Clark, C. R., Loveless, S., DeLosAngeles, D., Wallace, A. K., Broberg, M., and Willoughby, J. O. (2008). Thinking activates EMG in scalp electrical recordings. Clin. Neurophysiol. 119, 1166-1175.

Whitham, E. M., Pope, K. J., Fitzgibbon, S. P., Lewis, T., Clark, C. R., Loveless, S., Broberg, M., Wallace, A., DeLosAngeles, D., Lillie, P., Hardy, A., Fronsko, R., Pulbrook, A., and Willoughby, J. O. (2007).
Scalp electrical recording during paralysis: quantitative evidence that EEG frequencies above $20 \mathrm{~Hz}$ are contaminated by EMG. Clin. Neurophysiol. 118, 1877-1888.

Conflict of Interest Statement: The authors declare that the research was conducted in the absence of any commercial or financial relationships that could be construed as a potential conflict of interest.

Received: 02 August 2011; paper pending published: 26 October 2011; accepted: 21 November 2011; published online: 07 December 2011.
Citation: Whitham EM, Fitzgibbon SP, Lewis TW, Pope KJ, DeLosAngeles D, Clark CR, Lillie P, Hardy A, Gandevia SC and Willoughby JO (2011) Visual experiences during paralysis. Front. Hum. Neurosci. 5:160. doi: 10.3389/fnhum.2011.00160

Copyright () 2011 Whitham, Fitzgibbon, Lewis, Pope, DeLosAngeles, Clark, Lillie, Hardy, Gandevia and Willoughby. This is an open-access article distributed under the terms of the Creative Commons Attribution Non Commercial License, which permits non-commercial use, distribution, and reproduction in other forums, provided the original authors and source are credited. 